\title{
On the Role of Solute Solvation and Excluded-Volume Interactions in Coupled Diffusion
}

\author{
Onofrio Annunziata* \\ Department of Chemistry, Texas Christian University, Fort Worth, Texas 76129
}

Received: May 6, 2008; Revised Manuscript Received: July 16, 2008

\begin{abstract}
Coupled diffusion is observed in multicomponent liquid mixtures in which strong thermodynamic interactions occur. This phenomenon is described by cross terms in the matrix of multicomponent diffusion coefficients. This paper reports a theoretical analysis on the relative role of thermodynamic factors and Onsager crosscoefficients on cross-diffusion coefficients relevant to ternary mixtures containing macromolecules or colloidal particles in the presence of salting-out conditions. A new model based on frictional coefficients between solvated solutes is reported. This model predicts that the Onsager cross-coefficient is negative and contributes significantly to cross-diffusion coefficients even at infinite dilution for solutes with a large difference in size. These predictions are consistent with recent experimental results. The role of preferential solvation and excludedvolume interactions on the thermodynamic factors are also examined. Excluded-volume interactions are introduced through the use of the McMillan-Mayer thermodynamic framework after emphasizing some important aspects of diffusion reference frames and thermodynamic driving forces. Finally, new expressions for cross-diffusion coefficients are proposed.
\end{abstract}

\section{Introduction}

Diffusion plays an important role in many industrial, geochemical, and biochemical processes. ${ }^{1}$ Modeling these processes include the knowledge of the diffusion coefficients. ${ }^{2}$ The complete description of a system with $N$ solutes requires an $N$ $\times N$ matrix of diffusion coefficients, $D_{i j}$, relating the flux of each solute component to the gradients of all solute components. ${ }^{3}$ The $N$ diagonal main coefficients, $D_{i i}$, characterize the flux of a solute due to its own concentration gradient whereas the remaining $N(N-1)$ cross-coefficients, $D_{i j}$ with $i \neq j$, characterize the flux of a solute due to the concentration gradient of the other solute. These cross-terms describe coupled diffusion.

In many cases, diffusion processes are modeled with the simplification that the $N(N-1)$ cross-diffusion coefficients can be neglected. ${ }^{1,2}$ However, this approximation may not be valid for strongly interacting solutes or for processes occurring in the presence of large concentration gradients such as those at the interface between two different mixtures. Thus, it is generally important to measure and predict cross-diffusion coefficients. ${ }^{4}$

Since cross-diffusion coefficients describe the net interaction between two different solutes, experimental ${ }^{5-17}$ and theoretical $^{18-24}$ investigations on ternary systems have played a chief role in the comprehension of coupled diffusion. Moreover, measurements of ternary diffusion coefficients have been used to reliably extract thermodynamic data. $7,8,25$

For ternary systems with two electrolyte solutes sharing a common ion, the two cross-diffusion coefficients can be predicted at infinite dilution by using the Nernst-Hartley equations. ${ }^{3,18}$ These relations are based on the electrostatic coupling existing between ionic species. Expressions for the two cross-diffusion coefficients are also available for ternary systems where chemical association occurs between the solutes. ${ }^{20}$ However, coupled diffusion is not well-understood for ternary systems in which the two solutes exert a salting-out effect on one another and common-ion effects are absent. ${ }^{7,20}$

* Corresponding author. Phone: (817) 257-6215. (817) 257-5851. E-mail: O.Annunziata@tcu.edu.
This phenomenon becomes particularly interesting in the case of macromolecular solutions, where additives are often introduced to induce phase transitions by salting-out interactions. ${ }^{6}$ Coupled diffusion is also important for modeling the drying behavior of polymer-solvent mixtures. ${ }^{22}$

In most cases, prediction of $D_{i j}$ at infinite dilution with respect to both solutes is made through the approximation that the ratio $D_{i j} / D_{i i}$ is a thermodynamic factor. This can be either calculated from available thermodynamic data or estimated through the use of equilibrium models. ${ }^{9-12,20}$ This approximation is however based on the assumption that the frictional interaction between the two solutes can be neglected with respect to the corresponding thermodynamic interaction.

The assumption that $D_{i j} / D_{i i}$ is essentially a thermodynamic factor is questionable even at infinite dilution. Indeed, for ternary aqueous salt solutions containing a neutral macromolecule, it has been recently observed that only one of the two diffusioncoefficient ratios can be approximated as a thermodynamic factor. ${ }^{7}$ In contrast, the other diffusion-coefficient ratio was found to be significantly smaller than its corresponding thermodynamic factor. This implies that frictional interactions cannot be generally neglected. Thus, it becomes clearly important to understand in which conditions thermodynamic approximation becomes reliable. Models based on frictional coefficients ${ }^{20,22,24,26-28}$ can be used to characterize the frictional interaction between the two solutes. However, current approaches qualitatively fail to describe the observed discrepancy between diffusion-coefficient ratios and thermodynamic factors. ${ }^{7}$

The role of excluded-volume interactions on the thermodynamic factors have been examined also. ${ }^{6,9-12}$ For ternary systems, thermodynamic data on solute-solute interactions are often not available. Thus, thermodynamic factors are estimated through the use of models. For macromolecular solutions, it has been proposed that excluded-volume effects are mostly responsible for the experimentally observed large and positive values of $D_{i j} / D_{i i}{ }^{6}$ Hence, predictive equations based on excludedvolume effects have been reported for these systems. ${ }^{9-11}$ However, the thermodynamic basis and the actual presence of 
excluded-volume effects in $D_{i j} / D_{i i}$ have been questioned. ${ }^{12}$ Arguing along this line, it has been proposed that positive large $D_{i j} / D_{i i}$ can be obtained even for ideal solutions. Indeed, for ideal solutions, it can been shown that $D_{i j} / D_{i i}$ is directly proportional to the difference in partial molar volume between solute $j$ and solvent. ${ }^{12,20}$ This result however raises two concerns in relation to solution mixing entropy. First, the expression of ideal mixing entropy has been microscopically derived by using the hypothesis that the molecular volumes of all system components are the same. Thus, the derived expression for $D_{i j} / D_{i i}$ is expected to be accurate only in the limit that partial-molar-volume differences between solutes and solvent are small. Clearly, this condition does not apply to macromolecular solutions. Second, well-established expressions of mixing entropy based on excluded-volume interactions have been extensively employed for the thermodynamic characterization of macromolecular solutions or colloidal suspensions. ${ }^{29,30}$ Thus, it is expected that excluded-volume effects significantly contribute to the $D_{i j} / D_{i i}$ thermodynamic factor for macromolecular solutions.

The main objective of this paper is to theoretically examine the contribution of both frictional and thermodynamic terms in $D_{i j} / D_{i i}$ for ternary systems in which solute solvation and excluded-volume interactions are present. This paper is organized in the following way. In Section II, fundamental driving forces for diffusion are examined in the context of McMillanMayer theory ${ }^{31}$ in order to formally introduce excluded-volume interactions. The extraction of thermodynamic data from experimental ternary diffusion coefficients is also discussed in relation to excluded-volume interactions. In Section III, the frictional-coefficient formalism is reviewed and compared with recent experimental results. In Section IV, a new model for the frictional coefficients based on solute solvation consistent with experimental results is reported. In Section $\mathrm{V}$, the relative contribution of frictional and thermodynamic terms in $D_{i j} / D_{i i}$ is examined at infinite dilution based on solute solvation and excluded-volume interactions.

\section{Thermodynamic Driving Forces for Diffusion}

Isothermal diffusion is driven by the gradient of chemical potential, $\mu_{i}$, of the system components. According to nonequilibrium thermodynamics, ${ }^{32,33}$ the rate of entropy production $\sigma$ is related to $\nabla \mu_{i}$ and the molar fluxes, $J_{i}$, taken with respect to the center of mass of the system. For a system with $N+1$ components, we have

$$
-T \sigma=\sum_{i=0}^{N} J_{i} \nabla \mu_{i}
$$

where $T$ is the absolute temperature. Here, the subscript 0 is used to denote the solvent component.

We can introduce the fluxes, $\left(J_{i}\right)_{0}=J_{i}-\left(C_{i} / C_{0}\right) J_{0}$, taken with respect to the solvent frame of reference, where $C_{i}$ (with $i=0, \ldots, N)$ is the molar concentration of component $i^{3,21,34,35}$ If we insert $\left(J_{i}\right)_{0}$ into eq 1 , we obtain

$$
-T \sigma=\sum_{i=1}^{N}\left(J_{i}\right)_{0} \nabla \mu_{i}
$$

where the Gibbs-Duhem equation

$$
\sum_{i=0}^{N} C_{i} \nabla \mu_{i}=0
$$

has been applied. Because $\sigma$ is a sum of products of fluxes and forces, the following linear law holds, ${ }^{32,33}$

$$
-\left(J_{i}\right)_{0}=\sum_{j=1}^{N}\left(L_{i j}\right)_{0} \nabla \mu_{j} \quad(\text { with } i=1, \ldots, N)
$$

where the $\left(L_{i j}\right)_{0}$ are the solvent-frame Onsager coefficients. These coefficients obey the Onsager reciprocal relations: $\left(L_{i j}\right)_{0}=\left(L_{j i}\right)_{0}$ with $i \neq j .35,36$

We can also introduce the fluxes, $\left(J_{i}\right)_{V}=J_{i}-C_{i} \sum_{i=0}^{N} \bar{V}_{i} J_{i}$, taken with respect to the center of volume of the system, 3,35 where $\bar{V}_{i}$ (with $i=0, \ldots, N$ ) is the partial molar volume of component $i$. If we insert $\left(J_{i}\right)_{V}$ into eq 1 with $\bar{V}_{0}\left(J_{0}\right)_{V}=$ $-\sum_{i=1}^{N} \bar{V}_{i}\left(J_{i}\right)_{V}$, we obtain

$$
-T \sigma=\sum_{i=1}^{N}\left(J_{i}\right)_{V}\left[\nabla \mu_{i}-\left(\bar{V}_{i} / \bar{V}_{0}\right) \nabla \mu_{0}\right]
$$

According to eq 5, linear laws for the volume-frame fluxes are written by taking the forces, $\nabla \mu_{i}-\left(\bar{V}_{i} / \bar{V}_{0}\right) \nabla \mu_{0}$, instead of $\nabla \mu_{i}$

$$
-\left(J_{i}\right)_{V}=\sum_{j=1}^{N}\left(L_{i j}\right)_{V}\left[\nabla \mu_{i}-\left(\bar{V}_{i} / \bar{V}_{0}\right) \nabla \mu_{0}\right] \quad(\text { with } i=1, \ldots, N)
$$

where the $\left(L_{i j}\right)_{V}$ are the volume-frame Onsager coefficients and $\left(L_{i j}\right)_{V}=\left(L_{j i}\right)_{V}$ with $i \neq j$.

Diffusion processes are normally described with respect to concentration gradients through the use of Fick's first law ${ }^{3}$

$$
-\left(J_{i}\right)_{r}=\sum_{j=1}^{N}\left(D_{i j}\right)_{r} \nabla C_{j} \quad(\text { with } i=1, \ldots, N)
$$

where the $\left(D_{i j}\right)_{r}$ 's are the diffusion coefficients and the subscript $r$ denotes the reference frame (e.g., 0 or $V$ ). Diffusion coefficients can be expressed in terms of Onsager coefficients and thermodynamic factors by applying eqs 4, 6, and 7,

$$
\begin{gathered}
\left(D_{i j}\right)_{0}=\sum_{k=1}^{N}\left(L_{i k}\right)_{0} \mu_{k j} \quad(\text { with } i, j=1, \ldots, N) \\
\left(D_{i j}\right)_{V}=\sum_{k=1}^{N}\left(L_{i k}\right)_{V}\left[\mu_{k j}-\left(\bar{V}_{i} / \bar{V}_{0}\right) \mu_{0 j}\right] \quad(\text { with } i, j=1, \ldots, N)
\end{gathered}
$$

where the $\mu_{k j}=\left(\partial \mu_{k} / \partial C_{j}\right)_{C_{i}}, i \neq j$ are the thermodynamic factors characterizing the interactions between the solution components.

To an excellent approximation, measured diffusion coefficients correspond to the volume-fixed frame. ${ }^{34}$ However, the solvent-frame diffusion coefficients, $\left(D_{i j}\right)_{0}$, are more simply related to chemical-potential derivatives compared to the $\left(D_{i j}\right)_{V}$. Thus, thermodynamic analysis on diffusion data is usually performed on $\left(D_{i j}\right)_{0} \cdot{ }^{25}$ These diffusion parameters are calculated from the measured $\left(D_{i j}\right)_{V}$ by using 21,37

$$
\left(D_{i j}\right)_{0}=\left(D_{i j}\right)_{V}-C_{i} \sum_{k=1}^{N} \bar{V}_{k}\left(D_{k j}\right)_{V} \quad(\text { with } i, j=1, \ldots, N)
$$

However, as it will be now shown, it may be more convenient to perform thermodynamic analysis directly on the volumeframe thermodynamic factor, $\left[\mu_{k j}-\left(\bar{V}_{i} / \bar{V}_{0}\right) \mu_{0 j}\right]$, instead of $\mu_{k j}$, for macromolecular systems. This observation follows from the realization that chemical potentials of an incompressible liquid mixture with $N+1$ components can be formally converted into effective chemical potentials of a gas mixture with $N$ components, and the osmotic pressure of the liquid mixture is equivalent to the gas pressure. This is a well-known cor- 
respondence that relates to the McMillan-Mayer theory based on statistical mechanics. ${ }^{31,38-41}$ This conversion requires the assumption that the partial molar volumes and, consequently, the total volume are not changing during an isobaric and isothermal liquid diffusion process (see Appendix A). The effective chemical potentials are related to the real chemical potentials by ${ }^{40}$

$$
\tilde{\mu}_{i} \equiv \mu_{i}-\left(\bar{V}_{i} / \bar{V}_{0}\right) \mu_{0} \quad(\text { with } i=1, \ldots, N)
$$

Thus, volume-frame diffusion coefficients, $\left(D_{i j}\right)_{V}$, are directly related to the derivatives of $\tilde{\mu}_{i}$. By assuming that $\bar{V}_{i}$ is constant, eq 9 becomes

$$
\left(D_{i j}\right)_{V}=\sum_{k=1}^{N}\left(L_{i k}\right)_{V} \tilde{\mu}_{k j} \quad(\text { with } i, j=1, \ldots, N)
$$

where $\tilde{\mu}_{k j}=\left(\partial \tilde{\mu}_{k} / \partial C_{j}\right)_{C_{i}, i \neq j}$. One important property of $\tilde{\mu}_{k j}$, which does not generally apply to $\mu_{k j},{ }^{19,25}$ is that $\tilde{\mu}_{j k}=\tilde{\mu}_{k j}$ with $j \neq k$.

To examine the difference between $\tilde{\mu}_{k j}$ and $\mu_{k j}$, we will now consider their relation with the osmotic pressure, $\Pi$, through the Gibbs-Duhem equations:

$$
\begin{gathered}
\sum_{i=1}^{N} C_{i} d \tilde{\mu}_{i}=d \Pi \\
\sum_{i=1}^{N} C_{i} d \mu_{i}=-C_{0} d \mu_{0}=C_{0} \bar{V}_{0} d \Pi
\end{gathered}
$$

For dilute solutions, we can introduce the virial equation of state for a gas mixture according to the McMillan-Mayer theoretical framework

$$
\frac{\Pi}{R T}=C\left(1+C \sum_{k=1}^{N} \sum_{j=1}^{N} x_{k} x_{j} B_{k j}+\ldots\right)
$$

where $C=\sum_{i=1}^{N} C_{i}, x_{i}=C_{i} / C$, the $B_{k j}$ 's are the second virial coefficients, and $B_{k j}=B_{j k}$ with $k \neq j$. If solvent molecules are small compared to solute particles, statistical mechanics can be used to relate the $B_{k j}$ to a mean-force potential describing solvent-mediated interactions between the solute particles. ${ }^{31}$ Here, the solvent continuum plays the role that vacuum does in an actual gas system. It is through the use of $B_{k j}$ that excludedvolume interactions can be properly inserted in the chemicalpotential expressions.

We can now derive expressions for $\tilde{\mu}_{k j}$ and $\mu_{k j}$ in terms of $B_{k j}$ by using eqs $13-15$.

$$
\begin{gathered}
\tilde{\mu}_{i j} / R T=\delta_{i j} / C_{i}+2 B_{i j}+\ldots \\
\mu_{i j} / R T=\delta_{i j} / C_{i}+2 B_{i j}-\bar{V}_{i}+\ldots
\end{gathered}
$$

where $\delta_{i j}$ is the Kronecker delta. It is important to remark that $\tilde{\mu}_{i j}$ is directly related to virial coefficients and not $\mu_{i j}$. Because the distinction between $\tilde{\mu}_{i}$ and $\mu_{i}$ has not always been clearly made, ${ }^{42,43}$ confusion has arisen concerning the application of hard-sphere models to liquid mixtures. In Section V, relationships between cross-diffusion coefficients and hard-sphere models are obtained by using eqs 16 and 17 .

It has been previously shown ${ }^{25}$ that the chemical potential derivatives $\mu_{12}$ and $\mu_{21}$ can be extracted from experimental ternary diffusion coefficients. In Appendix B, it is shown how this approach can be extended to the extraction of $\tilde{\mu}_{12}$.

\section{Frictional-Coefficient Formulation of Diffusion}

The Onsager transport coefficients can be examined in term of frictional coefficients. Indeed, a diffusion process can be thought to occur in a quasi-stationary regime in which the thermodynamic driving forces equal the opposing frictional force. On the basis of frictional forces, the following linear laws for diffusion have been proposed by Bearman: ${ }^{27}$

$$
-\nabla \mu_{i}=\sum_{j=0}^{N} C_{j} \zeta_{i j}\left(u_{i}-u_{j}\right) \quad(\text { with } i, j=0, \ldots, N)
$$

where $\zeta_{i j}$ are coefficients describing the frictional force between species $i$ and species $j$, and $u_{i}$ is the species velocity of component $i$ relative to the center of mass of the system. The frictional coefficients satisfy the conditions $\sum_{j=0}^{N} C_{j} \zeta_{i j}=0$ and $\zeta_{i j}=\xi_{j i}$ with $i \neq j .{ }^{35}$ Equation 18 is equivalent to resistancebased equations previously reported by Onsager ${ }^{26}$ and to the Stefan-Maxwell equations. ${ }^{44}$ Contrary to $\left(L_{i k}\right)_{r}, \zeta_{i j}$ are frame independent and have a direct physical interpretation in terms of friction between the system components. ${ }^{45}$ Hence, several theoretical examinations of the diffusion coefficients start from frictional coefficients. In some models, the $\zeta_{i j}$ 's have been estimated by using the approximation $\zeta_{i j}{ }^{2}=\zeta_{i i} \zeta_{j j}$. ${ }^{22,24}$ Generally, all $\zeta_{i j}$ values (with $i \neq j$ ) are assumed to be positive, consistently with the physical concept of friction. However, this assumption is not a necessary condition for the second law of thermodynamics to be respected, and negative values of $\zeta_{i j}$ may occur in principle. This apparent discrepancy between thermodynamics and friction formalism can be understood by observing that eq 18 is defined with respect to the system components and not the actual diffusing species. Because of physical or chemical association between two components, new chemical species can be formed. If eq 18 were defined with respect to the diffusing species, all frictional-coefficient values may be positive. In Section IV, we will examine the sign of $\xi_{i j}$ in relation to solvated solute molecules.

The relation of the solvent-frame Onsager coefficients, $\left(L_{i j}\right)_{0}$, to $\zeta_{i j}$ will be now examined for a ternary system. Note that the volume-frame coefficients, $\left(L_{i j}\right)_{V}$, which are less straightforwardly related to the frictional coefficients, can be obtained from $\left(L_{i j}\right)_{0}$ by applying eq 3 and $\left(J_{i}\right)_{V}=\left(J_{i}\right)_{0}-C_{i} \sum_{i}^{N}={ }_{1} \bar{V}_{i}\left(J_{i}\right)_{0}$ to eqs 4 and 5 . This yields

$$
\begin{gathered}
\left(L_{11}\right)_{V}=\left(1-C_{1} \bar{V}_{1}\right)^{2}\left(L_{11}\right)_{0}-2 C_{1} \bar{V}_{2}\left(1-C_{1} \bar{V}_{1}\right)\left(L_{12}\right)_{0}+ \\
C_{1}^{2} \bar{V}_{2}^{2}\left(L_{22}\right)_{0}(19) \\
\left(L_{12}\right)_{V}=\left(1-C_{1} \bar{V}_{1}-C_{2} \bar{V}_{2}\right)\left(L_{12}\right)_{0}-C_{2} \bar{V}_{1}\left(1-C_{1} \bar{V}_{1}\right)\left(L_{11}\right)_{0}- \\
C_{1} \bar{V}_{2}\left(1-C_{2} \bar{V}_{2}\right)\left(L_{22}\right)_{0}(20) \\
\left(L_{22}\right)_{V}=\left(1-C_{2} \bar{V}_{2}\right)^{2}\left(L_{22}\right)_{0}-2 C_{2} \bar{V}_{1}\left(1-C_{2} \bar{V}_{2}\right)\left(L_{12}\right)_{0}+ \\
C_{2}^{2} \bar{V}_{1}^{2}\left(L_{11}\right)_{0}(21)
\end{gathered}
$$

For a ternary system, eq 18 reduces to

$$
\begin{aligned}
& \nabla \mu_{1}=C_{0} \zeta_{10}\left(u_{0}-u_{1}\right)+C_{2} \zeta_{12}\left(u_{2}-u_{1}\right) \\
& \nabla \mu_{2}=C_{0} \zeta_{20}\left(u_{0}-u_{2}\right)+C_{1} \zeta_{21}\left(u_{1}-u_{2}\right)
\end{aligned}
$$

where $\zeta_{12}=\zeta_{21}$. Here, the expression for $\nabla \mu_{0}$ has been omitted because it can be recovered from eqs $22 \mathrm{a}, \mathrm{b}$ by using eq 3 . If $u_{i}$ $-u_{j}$ is replaced with $\left(J_{i}\right)_{0} / C_{i}-\left(J_{j}\right)_{0} / C_{j}$ in eqs $22 \mathrm{a}, \mathrm{b}$, we find 


$$
\begin{aligned}
& -C_{1} \nabla \mu_{1}=\left(C_{0} \zeta_{10}+C_{2} \zeta_{12}\right)\left(J_{1}\right)_{0}-C_{1} \zeta_{12}\left(J_{2}\right)_{0} \\
& -C_{2} \nabla \mu_{2}=\left(C_{0} \zeta_{20}+C_{1} \zeta_{12}\right)\left(J_{2}\right)_{0}-C_{2} \zeta_{21}\left(J_{1}\right)_{0}
\end{aligned}
$$

By comparing eqs $23 \mathrm{a}, \mathrm{b}$ with eq 4 , the following expressions for $\left(L_{i j}\right)_{0}$ are obtained: ${ }^{3}$

$$
\begin{gathered}
\left(L_{11}\right)_{0}=C_{1} \frac{C_{0} \zeta_{20}+C_{1} \zeta_{12}}{C_{0}^{2} \zeta_{10} \zeta_{20}+C_{0} \zeta_{12}\left(C_{1} \zeta_{10}+C_{2} \zeta_{20}\right)} \\
\left(L_{12}\right)_{0}=\left(L_{21}\right)_{0}=C_{1} C_{2} \frac{\zeta_{12}}{C_{0}^{2} \zeta_{10} \zeta_{20}+C_{0} \zeta_{12}\left(C_{1} \zeta_{10}+C_{2} \zeta_{20}\right)} \\
\left(L_{22}\right)_{0}=C_{2} \frac{C_{0} \zeta_{10}+C_{2} \zeta_{12}}{C_{0}^{2} \zeta_{10} \zeta_{20}+C_{0} \zeta_{12}\left(C_{1} \zeta_{10}+C_{2} \zeta_{20}\right)}
\end{gathered}
$$

Physical insight on the behavior of both $\left(L_{i j}\right)_{0}$ and $\left(L_{i j}\right)_{V}$ can be obtained by examining eqs $19-21$ and $24-26$ for dilute solutions. In this limiting case, we find

$$
\begin{gathered}
\left(L_{11}\right)_{0}=\left(L_{11}\right)_{V}=C_{1}\left(V_{0}^{*} / \zeta_{10}\right)=C_{1} D_{1}^{*} \\
\left(L_{22}\right)_{0}=\left(L_{22}\right)_{V}=C_{2}\left(V_{0}^{*} / \zeta_{20}\right)=C_{2} D_{2}^{*} \\
\left(L_{12}\right)_{0}=C_{1} C_{2}\left(V_{0}^{*} / \zeta_{10}\right)\left(V_{0}^{*} / \zeta_{20}\right) \xi_{12}=C_{1} C_{2} D_{1}^{*} D_{2}^{*} \zeta_{12} \\
\left(L_{12}\right)_{V}=\left(L_{12}\right)_{0}-C_{1} C_{2}\left(\bar{V}_{1} D_{1}^{*}+\bar{V}_{2} D_{2}^{*}\right)= \\
C_{1} C_{2}\left[D_{1}^{*} D_{2}^{*} \zeta_{12}-\right. \\
\left.\left(\bar{V}_{1} D_{1}^{*}+\bar{V}_{2} D_{2}^{*}\right)\right]
\end{gathered}
$$

where $V_{0}^{*}=1 / C_{0}$ is the molar volume of pure solvent and $D_{i}^{*}=$ $V_{0}^{*} / \zeta_{0 i}$ is the tracer diffusion coefficient of solute $i$. Equations 27-30 describe the first-order dependence of the Onsager coefficients on the solute concentrations. It is important to observe that $\left(L_{i i}\right)_{0}=\left(L_{i i}\right)_{V}$ to first order in $C_{i}$, whereas generally, the cross-terms $\left(L_{12}\right)_{0}$ and $\left(L_{12}\right)_{V}$ are not equal to each other to first-order in $C_{1} C_{2}$. Specifically, we can see that $\left(L_{12}\right)_{V}<\left(L_{12}\right)_{0}$. If we assume that $\zeta_{12}=0$, we obtain $\left(L_{12}\right)_{0}=0$ and $\left(L_{12}\right)_{V}=$ $-C_{1} C_{2}\left(\bar{V}_{1} D_{1}^{*}+\bar{V}_{2} D_{2}^{*}\right)$. One important observation related to this analysis is that neglecting $\left(L_{12}\right)_{0}$ in the expression of $\left(D_{i j}\right)_{0}$ does not necessarily imply that $\left(L_{12}\right)_{V}$ can be neglected in the expression of $\left(D_{i j}\right)_{V}$.

\section{Frictional Coefficients for Solvated Solutes}

We now consider the role of solute solvation on frictional coefficients. Although it is well established that solute molecules move together with their solvation shell in solution, ${ }^{3,46}$ this aspect has not been previously examined in relation to frictional coefficients. Because of solvent binding, the chemical potentials of the solvated solutes are

$$
\begin{aligned}
& \hat{\mu}_{1}=\mu_{1}+v_{1} \mu_{0} \\
& \hat{\mu}_{2}=\mu_{2}+v_{2} \mu_{0}
\end{aligned}
$$

where $v_{i}$ is the number of solvent molecules bound to solute $i$. On the basis of the frictional-coefficient formalism of eqs 22a,b, we can write the following expressions for the solvated solutes:

$$
\begin{aligned}
& \nabla \hat{\mu}_{1}=\hat{C}_{0} \hat{\zeta}_{10}\left(\hat{u}_{0}-u_{1}\right)+C_{2} \hat{\zeta}_{12}\left(u_{2}-u_{1}\right) \\
& \nabla \hat{\mu}_{2}=\hat{C}_{0} \hat{\zeta}_{20}\left(\hat{u}_{0}-u_{2}\right)+C_{1} \hat{\zeta}_{21}\left(u_{1}-u_{2}\right)
\end{aligned}
$$

where $\nabla \hat{\mu}_{i}=\nabla \mu_{i}+v_{i} \nabla \mu_{0}, \hat{\zeta}_{i 0}$ describes the frictional force between solvated solute $i$ and free solvent, and $\hat{\zeta}_{12}$ describes that between the two solvated solutes. In eqs 32a,b, $\hat{C}_{0}=C_{0}$ $-v_{1} C_{1}-v_{2} C_{2}$ is the concentration of free solvent and $\hat{u}_{0}$ is the velocity of the free-solvent molecules relative to the center of mass of the system. It is important to observe that $\nabla \hat{\mu}_{i}$ are the appropriate thermodynamic driving forces for the fluxes taken with respect to the free-solvent frame of reference (see Appendix C).

The relation between $\zeta_{i j}$ and $\hat{\zeta}_{i j}$ will be now derived. Because the free-solvent flux is given by $\hat{J}_{0}=J_{0}-v_{1} J_{1}-v_{2} J_{2}$, the following relation between $\hat{u}_{0}$ and $u_{0}$ can be obtained:

$$
\hat{u}_{0}=\frac{C_{0} u_{0}-v_{1} C_{1} u_{1}-v_{2} C_{2} u_{2}}{C_{0}-v_{1} C_{1}-v_{2} C_{2}}
$$

Equation 33 represents the difference between the free-solvent and solvent reference frames. If we insert eq 33 into eqs 32a,b and use eq 3 , we obtain

$$
\begin{array}{r}
{\left[\left(C_{0}-v_{1} C_{1}\right) \nabla \mu_{1}-v_{1} C_{2} \nabla \mu_{2}\right] / C_{0}=C_{0} \hat{\zeta}_{10}\left(u_{0}-u_{1}\right)+} \\
C_{2}\left(\hat{\zeta}_{12}-v_{2} \hat{\zeta}_{10}\right)\left(u_{2}-u_{1}\right) \\
{\left[\left(C_{0}-v_{2} C_{2}\right) \nabla \mu_{2}-v_{2} C_{1} \nabla \mu_{1}\right] / C_{0}=C_{0} \hat{\zeta}_{20}\left(u_{0}-u_{2}\right)+} \\
C_{1}\left(\hat{\zeta}_{21}-v_{1} \hat{\zeta}_{20}\right)\left(u_{1}-u_{2}\right)
\end{array}
$$

Equations 34a,b can be used to obtain explicit expressions for $\nabla \mu_{1}$ and $\nabla \mu_{2}$. The comparison with eqs 22a,b yields

$$
\begin{gathered}
\zeta_{10}=\frac{\left(C_{0}-v_{2} C_{2}\right) \hat{\zeta}_{10}+v_{1} C_{2} \hat{\zeta}_{20}}{C_{0}-v_{1} C_{1}-v_{2} C_{2}} \\
\xi_{20}=\frac{\left(C_{0}-v_{1} C_{1}\right) \hat{\zeta}_{20}+v_{2} C_{1} \hat{\zeta}_{10}}{C_{0}-v_{1} C_{1}-v_{2} C_{2}} \\
\xi_{12}=\hat{\zeta}_{12}-\frac{v_{2}\left(C_{0}-v_{2} C_{2}\right) \hat{\zeta}_{10}+v_{1}\left(C_{0}-v_{1} C_{1}\right) \hat{\zeta}_{20}}{C_{0}-v_{1} C_{1}-v_{2} C_{2}}
\end{gathered}
$$

For dilute solutions, we obtain the following relations between the Onsager transport coefficients and $\hat{\zeta}_{i j}$ :

$$
\begin{gathered}
\left(L_{11}\right)_{0}=\left(L_{11}\right)_{V}=C_{1}\left(V_{0}^{*} / \hat{\zeta}_{10}\right)=C_{1} D_{1}^{*} \\
\left(L_{22}\right)_{0}=\left(L_{22}\right)_{V}=C_{2}\left(V_{0}^{*} / \hat{\zeta}_{20}\right)=C_{2} D_{2}^{*} \\
\left(L_{12}\right)_{0}=C_{1} C_{2}\left[D_{1}^{*} D_{2}^{*} \hat{\zeta}_{12}-V_{0}^{*}\left(v_{1} D_{1}^{*}+v_{2} D_{2}^{*}\right)\right] \\
\left(L_{12}\right)_{V}=C_{1} C_{2}\left[D_{1}^{*} D_{2}^{*} \hat{\zeta}_{12}-\left(\bar{V}_{1}+v_{1} V_{0}^{*}\right) D_{1}^{*}-\left(\bar{V}_{2}+v_{2} V_{0}^{*}\right) D_{2}^{*}\right]
\end{gathered}
$$

We note that $\zeta_{i 0}=\hat{\zeta}_{i 0}$ in the limit of dilute solutions. Here, $\xi_{i 0}$ and $\hat{\zeta}_{i 0}$ describe tracer diffusion of the solutes independently of their solvation. Equation 40 shows that negative values of $\xi_{12}$ can be obtained in the presence of significant solute solvation.

For ternary mixtures in which the two solutes associate in solution, it can be shown that $\xi_{12}>0$ and, consequently, $\left(L_{12}\right)_{0}$ $>0 .{ }^{20}$ However, the behavior of $\xi_{12}$ for the case of two solutes exerting a salting-out effect on one another is not wellunderstood. Recently, diffusion coefficients were reported on the poly(ethylene glycol)(1)- $\mathrm{KCl}(2)$ - water(0) ternary system. ${ }^{7}$ Poly(ethylene glycol) is a neutral hydrophilic polymer for which salts are known to be salting-out agents. These diffusion data lead to $\left(L_{12}\right)_{0}<0$, thereby implying that $\zeta_{12}<0$. It can be shown that selective association of either the anion or the cation to the polymer leads to $\xi_{12}>0$. Hence, the ionic nature of one of the two solutes may not be responsible for this experimental result. For this system, it does not appear that the diffusion data 
can be simply interpreted in terms of the frictional coefficients described by eq 29 . On the other hand, the proposed solvation model for frictional coefficients is consistent with negative values of $\xi_{12}$. For solvated polymers with high molecular weight, $v_{i}$ may become large. Indeed, poly(ethylene glycol) undergoes extensive hydration in aqueous solutions. ${ }^{7,10,11}$ This implies that $\hat{\zeta}_{12}$ may be neglected in eq 37 and $\xi_{12} \approx-\left(v_{2} \hat{\zeta}_{10}+v_{1} \hat{\xi}_{20}\right)$.

\section{Behavior of Cross-Diffusion Coefficients}

Solute solvation and excluded-volume interactions can significantly affect coupled diffusion in solution. In this section, the role of thermodynamic factors and Onsager coefficients on the behavior of $\left(D_{i j}\right)_{0} /\left(D_{i i}\right)_{0}$ and $\left(D_{i j}\right)_{V} /\left(D_{i i}\right)_{V}$ is examined. For a ternary system, eq 8 yields the following expressions for the solvent-frame diffusion coefficients:

$$
\begin{aligned}
& \frac{\left(D_{12}\right)_{0}}{\left(D_{11}\right)_{0}}=\frac{\left(L_{11}\right)_{0} \mu_{12}+\left(L_{12}\right)_{0} u_{22}}{\left(L_{11}\right)_{0} u_{11}+\left(L_{12}\right)_{0} u_{21}} \\
& \frac{\left(D_{21}\right)_{0}}{\left(D_{22}\right)_{0}}=\frac{\left(L_{22}\right)_{0} u_{21}+\left(L_{12}\right)_{0} \mu_{11}}{\left(L_{22}\right)_{0} u_{22}+\left(L_{12}\right)_{0} u_{12}}
\end{aligned}
$$

By convention, the larger and smaller solutes will be labeled with 1 and 2, respectively. If the Onsager cross-coefficient, $\left(L_{12}\right)_{0}$, is zero in eqs $42 \mathrm{a}$,b, the ratios $\left(D_{i j}\right)_{0} /\left(D_{i i}\right)_{0}$ become equal to the corresponding thermodynamic factors, $\mu_{i j} / \mu_{i i}$. This assumption has led to the examination of $\left(D_{i j}\right)_{0} /\left(D_{i i}\right)_{0}$ strictly in terms of thermodynamic parameters. However, it has been experimentally observed for the poly(ethylene glycol)(1)$\mathrm{KCl}(2)$-water $(0)$ ternary system that only $\left(D_{21}\right)_{0} /\left(D_{22}\right)_{0} \approx \mu_{21} /$ $\mu_{22}$ is a valid approximation. Indeed, the ratio $\left(D_{12}\right)_{0} /\left(D_{11}\right)_{0}$ was found to be significantly smaller than $\mu_{12} / \mu_{11}{ }^{7}$ This implies that the thermodynamic approximation is valid only for the small solute $(i=2)$.

To discuss the validity of the thermodynamic approximation, the $\left(D_{i j}\right)_{0} /\left(D_{i i}\right)_{0}$ ratios will be examined in the limit of dilute solutions. Equation 40 will be used with the assumption that $\hat{\zeta}_{12}=0$. Because $\left(D_{i j}\right)_{0} /\left(D_{i i}\right)_{0}$ is directly proportional to $C_{i}$ and is independent of $C_{j}$ to first order, the magnitude of the quotients $\left(D_{i j}\right)_{0} /\left[C_{i}\left(D_{i i}\right)_{0}\right]$ will be considered. It is worth mentioning that the dilute-solution behavior of $\left(D_{i j}\right)_{0} /\left(D_{i i}\right)_{0}$ is approximately maintained even in concentrated solutions. ${ }^{7,10,11}$

In the limit of dilute solutions, $\left(D_{i i}\right)_{0}=\left(L_{i i}\right)_{0} \mu_{i i}$ and $\mu_{i i} / \mu_{j j}=$ $C_{j} / C_{i}$. Thus, eqs $42 \mathrm{a}, \mathrm{b}$ becomes

$$
\begin{gathered}
\frac{\left(D_{12}\right)_{0}}{C_{1}\left(D_{11}\right)_{0}}=\frac{\mu_{12}}{C_{1} \mu_{11}}+\frac{\left(L_{12}\right)_{0}}{C_{2}\left(L_{11}\right)_{0}} \\
\frac{\left(D_{21}\right)_{0}}{C_{2}\left(D_{22}\right)_{0}}=\frac{\mu_{21}}{C_{2} \mu_{22}}+\frac{\left(L_{12}\right)_{0}}{C_{2}\left(L_{22}\right)_{0}}
\end{gathered}
$$

The Onsager terms $\left(L_{i j}\right)_{0} /\left[C_{i}\left(L_{i i}\right)_{0}\right]$ in eqs $43 \mathrm{a}$,b describe the deviation of the diffusion quotients $\left(D_{i j}\right)_{0} /\left[C_{i}\left(D_{i i}\right)_{0}\right]$ from the corresponding thermodynamic quotients $\mu_{i j} /\left[C_{i} \mu_{i i}\right]$ in the limit of dilute solutions.

To evaluate the magnitude of the thermodynamic quotients, eq 17 can be used to obtain $\mu_{i j} /\left[C_{i} \mu_{i i}\right]=2 B_{12}-\bar{V}_{i}$. For the special case of a hard-sphere mixture, $\bar{V}_{i}=N_{\mathrm{A}}(4 / 3) \pi R_{i}{ }^{3}$ and the excluded-volume interactions are described by $B_{12}=N_{\mathrm{A}}(2 /$ 3) $\pi\left(R_{1}+R_{2}\right)^{3},{ }^{37}$ where $N_{\mathrm{A}}$ is the Avogadro's number and $R_{i}$ is the radius of component $i$. Figure 1 graphically illustrates the magnitude of $2 B_{12}$ compared to that of $\mu_{12} /\left[C_{1} \mu_{11}\right]$ and $\mu_{21} /$ $\left[C_{2} \mu_{22}\right]$ in the case of hard spheres. If $R_{2} / R_{1} \ll 1$, we obtain $2 B_{12}$ $=\bar{V}_{1}$. This limiting result is valid also for nonspherical solutes, provided that solute 1 is significantly larger than solute 2 . A
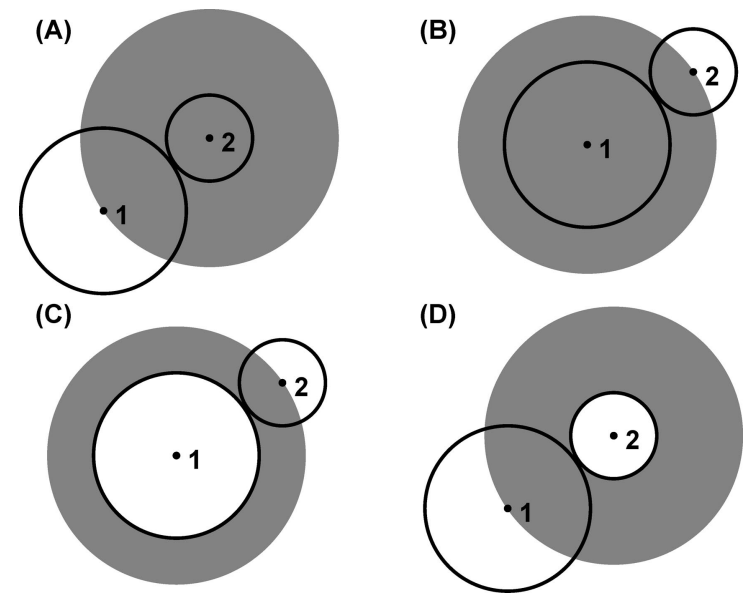

Figure 1. Graphic representation of hard-sphere excluded-volume interactions. The gray areas represent $2 B_{12}=\tilde{\mu}_{12} /\left(C_{1} \tilde{\mu}_{11}\right)$ $(A), 2 B_{12}=\widetilde{\mu}_{21} /\left(C_{1} \widetilde{\mu}_{22}\right)(\mathrm{B}), 2 B_{12}-\bar{V}_{1}=\mu_{12} /\left(C_{1} \mu_{11}\right)(\mathrm{C})$, and $2 B_{12}-$ $\bar{V}_{2}=\mu_{21} /\left(C_{2} \mu_{22}\right)$ (D). Note that $\tilde{\mu}_{12}=\tilde{\mu}_{21}$ and $\mu_{21} /\left(C_{2} \mu_{22}\right)-$ $\mu_{12} /\left(C_{1} \mu_{11}\right)=\bar{V}_{1}-\bar{V}_{2}$. In the limit of $R_{2} \ll R_{1}$, we obtain $2 B_{12}=\bar{V}_{1}$, $\mu_{12} /\left(C_{1} \mu_{11}\right)=0$, and $\mu_{21} /\left(C_{2} \mu_{22}\right)=\bar{V}_{1}$.

ternary system with $2 B_{12}=\bar{V}_{1}$ is a suitable reference system for ternary mixtures containing at least one macromolecular solute. Deviations from this reference system can be describe by introducing the phenomenological parameter $q$ defined by

$$
2 B_{12}=\bar{V}_{1}(1+q)^{3}
$$

where $q=R_{2} / R_{1}$ for the special case of a pure hard-sphere mixtures. Here, $q$ describes the thickness of a shell surrounding the large sphere and not accessible to the center of the small sphere (see gray layer in Figure 1c).

In general, $q$ does not necessarily characterize the relative size of the two solutes. For instance, $q$ can also be used to describe preferential solvation of macromolecules in the presence of small osmolytes. Indeed, macromolecule(1)-osmolyte(2) - solvent( 0 ) ternary systems can be modeled invoking the presence of a local domain represented by the solvent layer surrounding a given macromolecule. ${ }^{48-51}$ This domain is in equilibrium with a bulk domain characterizing the solvent-osmolyte remaining solution. Within this model, $\bar{V}_{1}\left[(1+q)^{3}-1\right]$ represents the excess of solvent volume in the local domain, whereas $\bar{V}_{1}(1+q)^{3}$ describes the volume excluded to the osmolyte because of the presence of the macromolecule and the solvation layer.

Explicit expressions for $\left(D_{i j}\right)_{0} /\left[C_{i}\left(D_{i i}\right)_{0}\right]$ can be obtained from eqs $43 \mathrm{a}$, b by applying eq 44 and eqs $38-40$ with $\hat{\zeta}_{12}=0$ :

$$
\frac{\left(D_{12}\right)_{0}}{C_{1}\left(D_{11}\right)_{0}}=\bar{V}_{1}\left[(1+q)^{3}-1\right]-v_{1} V_{0}^{*}\left[1+\left(v_{2} / v_{1}\right)\left(D_{2}^{*} / D_{1}^{*}\right)\right]
$$

$$
\begin{array}{r}
\frac{\left(D_{21}\right)_{0}}{C_{2}\left(D_{22}\right)_{0}}=\bar{V}_{1}\left[(1+q)^{3}-\left(\bar{V}_{2} / \bar{V}_{1}\right)\right]-v_{1} V_{0}^{*}\left[\left(D_{1}^{*} / D_{2}^{*}\right)+\right. \\
\left.\left(v_{2} / v_{1}\right)\right]
\end{array}
$$

To qualitatively examine the contribution of the Onsager terms compared to the corresponding thermodynamic terms, the quotients $-\left[\left(L_{12}\right)_{0} /\left(L_{11}\right)_{0}\right] /\left(\mu_{12} / \mu_{11}\right)$ and $-\left[\left(L_{12}\right)_{0} /\left(L_{22}\right)_{0}\right] /\left(\mu_{21} / \mu_{22}\right)$ are plotted as a function of $D_{1}^{*} / D_{2}^{*}$ in Figure 2. These quotients were calculated by using eqs $38-40$ and 44 for the special case of solvated spherical solutes with $v_{1} V_{0}^{*} / \bar{V}_{1}=0.1$. From this figure, it is important to observe that $-\left(L_{12}\right)_{0} /\left(L_{11}\right)_{0}$ becomes 


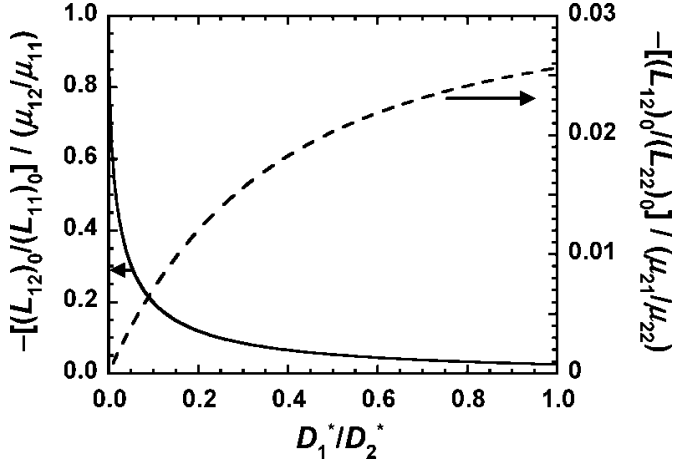

Figure 2. Plots of the solvent-frame quotients $-\left[\left(L_{12}\right)_{0} /\left(L_{11}\right)_{0}\right] /\left(\mu_{12} /\right.$ $\left.\mu_{11}\right)$ (solid curve) and $-\left[\left(L_{12}\right)_{0} /\left(L_{22}\right)_{0}\right] /\left(\mu_{21} / \mu_{22}\right)$ (dashed curve) as a function of $D_{1}^{*} / D_{2}^{*}$. These quotients were calculated by using eqs $38-40$ and 44 for the special case of solvated spherical solutes with $\nu_{1} V_{0}^{*} / \bar{V}_{1}$ $=0.1,\left(\bar{V}_{2} / \bar{V}_{1}\right)=\left(D_{1}^{*} / D_{2}^{*}\right)^{3}$, and $\left(v_{2} / v_{1}\right)=\left(D_{1}^{*} / D_{2}^{*}\right)^{2}$. The factor $q$ was calculated by using the expression $q=\left(R_{2}+\delta_{1}+\delta_{2}\right) / R_{1}$, where $\delta_{1}$ and $\delta_{2}$ are the thickness of the solvation layers surrounding the spherical solutes, $R_{2} / R_{1}=\left(\bar{V}_{2} / \bar{V}_{1}\right)^{1 / 3}, \delta_{1} / R_{1}=\left[1+\left(v_{1} V_{0}^{*} / \bar{V}_{1}\right)\right]^{1 / 3}-1$, and $\delta_{2} / R_{2}=$ $\left[1+\left(v_{2} / v_{1}\right)\left(\bar{V}_{1} / \bar{V}_{2}\right)\left(v_{1} V_{0}^{*} / \bar{V}_{1}\right)\right]^{1 / 3}-1$.

comparable to $\mu_{12} / \mu_{11}$ for two solvated solutes with a very large difference in size. Correspondingly, $-\left(L_{12}\right)_{0} /\left(L_{22}\right)_{0}$ becomes negligible with respect to $\mu_{21} / \mu_{22}$. If the two solutes have comparable sizes, it becomes reasonable to approximate both diffusion quotients with the corresponding thermodynamic factors. Experimental investigations on Poly(ethylene glycol $)^{7}$ and lysozyme ${ }^{8}$ in the presence of aqueous $\mathrm{KCl}$ correspond to $D_{1}^{*} / D_{2}^{*}$ equal to 0.03 and 0.06 respectively. Clearly, $-\left(L_{12}\right)_{0} /$ $\left(L_{11}\right)_{0}$ cannot be neglected for these systems.

To further examine the case of two solvated solutes with a very large difference in size, the conditions: $D_{1}^{*} / D_{2}^{*} \ll 1, \bar{V}_{2} / \bar{V}_{1}$ $\ll 1$ and $v_{2} / \nu_{1} \ll 1$ are applied to eqs $45 \mathrm{a}, \mathrm{b}$. Furthermore, because the diffusion coefficient is expected to have the weakest dependence on solute size, $v_{2} / v_{1} \ll D_{1}^{*} / D_{2}^{*}$ in eq 45 a can be also assumed. Thus, eqs $45 \mathrm{a}, \mathrm{b}$ become

$$
\begin{gathered}
\frac{\left(D_{12}\right)_{0}}{C_{1}\left(D_{11}\right)_{0}}=\bar{V}_{1}(1+q)^{3}-\left(\bar{V}_{1}+v_{1} V_{0}^{*}\right) \\
\frac{\left(D_{21}\right)_{0}}{C_{2}\left(D_{22}\right)_{0}}=\bar{V}_{1}(1+q)^{3}
\end{gathered}
$$

According to these limiting equations, $\left(D_{21}\right)_{0} /\left[C_{2}\left(D_{22}\right)_{0}\right]$ is a thermodynamic factor. Indeed, eq $46 \mathrm{~b}$ has been used to describe excluded-volume effects of solvated macromolecules on small osmolytes. ${ }^{8}$ Here, the solvated macromolecules can be regarded as a second phase that excludes volume to the osmolyte. Increasing the concentration of macromolecules at constant osmolyte concentration will increase the effective concentration of osmolyte in the solution between solvated macromolecules. Given a uniform concentration of osmolyte in a gradient of macromolecule concentration, there will be an effective concentration gradient of osmolyte that is directly proportional to macromolecule concentration gradient. This in turn will drive a flux of osmolyte from higher to lower macromolecule concentration regions, which will be reflected in a positive value of $\left(D_{21}\right)_{0}$ according to eq $46 \mathrm{~b}$. However, according to eq $46 \mathrm{a}$, this excluded-volume interpretation cannot be extended to $\left(D_{12}\right)_{0} /$ $\left[C_{1}\left(D_{11}\right)_{0}\right]$ because of the significant contribution of $\left(\bar{V}_{1}+v_{1} V_{0}^{*}\right)$, which can be regarded as the hydrodynamic volume ${ }^{46}$ of the large solute. Hence, $\left(D_{12}\right)_{0} /\left[C_{1}\left(D_{11}\right)_{0}\right]$ is given by the difference between excluded and hydrodynamic volume. Because it is reasonable to expect that these two volumes are similar in magnitude, we can deduce that $-\left(L_{12}\right)_{0} /\left(L_{11}\right)_{0} \approx \mu_{12} / \mu_{22}$. This property has been indeed observed for the poly(ethylene glycol)(1)- $\mathrm{KCl}(2)-$ water(0) ternary system. ${ }^{7}$

The limiting expressions of $\left(D_{i j}\right)_{V} /\left[C_{i}\left(D_{i i}\right)_{V}\right]$ differ from those of $\left(D_{i j}\right)_{0} /\left[C_{i}\left(D_{i i}\right)_{0}\right]$ because the cross-terms $\left(L_{12}\right)_{0}$ and $\left(L_{12}\right)_{V}$ are not equal to each other even at infinite dilution. For a ternary system, eq 9 yields the following expressions for the volumeframe diffusion coefficients:

$$
\begin{array}{r}
\frac{\left(D_{12}\right)_{V}}{C_{1}\left(D_{11}\right)_{V}}=\frac{\tilde{\mu}_{12}}{C_{1} \tilde{\mu}_{11}}+\frac{\left(L_{12}\right)_{V}}{C_{1} C_{2} D_{1}^{*}}=\bar{V}_{1}(1+q)^{3}- \\
{\left[\left(\bar{V}_{1}+v_{1} V_{0}^{*}\right)+\left(\bar{V}_{2}+v_{2} V_{0}^{*}\right)\left(D_{2}^{*} / D_{1}^{*}\right)\right]} \\
\frac{\left(D_{21}\right)_{V}}{C_{2}\left(D_{22}\right)_{V}}=\frac{\tilde{\mu}_{21}}{C_{2} \tilde{\mu}_{22}}+\frac{\left(L_{21}\right)_{V}}{C_{1} C_{2} D_{2}^{*}}=\bar{V}_{1}(1+q)^{3}- \\
{\left[\left(\bar{V}_{1}+v_{1} V_{0}^{*}\right)\left(D_{1}^{*} / D_{2}^{*}\right)+\left(\bar{V}_{2}+v_{2} V_{0}^{*}\right)\right]}
\end{array}
$$

It is important to observe that both expressions contain the same thermodynamic factor $\bar{V}_{1}(1+q)^{3}$. Excluded-volume interactions between solutes may prevail with respect to solvation for the case of solute particles significantly larger than the solvent molecules. In this limit, the surface-to-volume ratio of the solute particles may be small, and the volume of bound solvent may be negligible compared to the intrinsic volume of the particles. This condition may approximately apply to spherical colloidal particles (e.g., large micelles and inorganic nanoparticles) and very large globular proteins. This condition may however not apply to solvated polymer coils for which the ratio of $v_{i} V_{0}^{*}$ to $\bar{V}_{i}$ may be independent of polymer size. If $\bar{V}_{i} \gg v_{i} V_{0}^{*}$, we can write

$$
\begin{aligned}
& \frac{\left(D_{12}\right)_{V}}{C_{1}\left(D_{11}\right)_{V}}=\bar{V}_{1}(1+q)^{3}-\bar{V}_{1}-\bar{V}_{2}\left(D_{2}^{*} / D_{1}^{*}\right) \\
& \frac{\left(D_{21}\right)_{V}}{C_{2}\left(D_{22}\right)_{V}}=\bar{V}_{1}(1+q)^{3}-\bar{V}_{1}\left(D_{1}^{*} / D_{2}^{*}\right)-\bar{V}_{2}
\end{aligned}
$$

It is important to observe that the Onsager terms in eqs 48a,b cannot be ignored even if $v_{i}=0$ because of the offset between $\left(L_{12}\right)_{0}$ and $\left(L_{12}\right)_{V}$. For a pure hard-sphere mixture, we can substitute $q=R_{2} / R_{1}=D_{1}^{*} / D_{2}^{*}=\left(\bar{V}_{2} / \bar{V}_{1}\right)^{1 / 3}$ in eqs $48 \mathrm{a}$,b. This allows us to obtain the following expressions for $\left(D_{i j}\right)_{V} /\left[C_{i}\left(D_{i i}\right)_{V}\right]$ :

$$
\begin{aligned}
& \frac{\left(D_{12}\right)_{V}}{C_{1}\left(D_{11}\right)_{V}}=\bar{V}_{1}\left(3+2 q+q^{2}\right) q \\
& \frac{\left(D_{21}\right)_{V}}{C_{2}\left(D_{22}\right)_{V}}=\bar{V}_{1}\left(1+2 q+3 q^{2}\right)
\end{aligned}
$$

These expressions describe pure depletion interactions between the two spherical solutes. In the limiting case of two solutes with a very large difference in size, we obtain $\left(D_{12}\right)_{V} /$ $\left[C_{1}\left(D_{11}\right)_{V}\right]=0$ and $\left(D_{21}\right)_{V} /\left[C_{2}\left(D_{22}\right)_{V}\right]=\bar{V}_{1}$.

\section{Summary and Conclusions}

The roles of solute solvation and excluded-volume interactions on $\left(D_{i j}\right)_{0} /\left[C_{i}\left(D_{i i}\right)_{0}\right]$ and $\left(D_{i j}\right)_{V} /\left[C_{i}\left(D_{i i}\right)_{V}\right]$ have been theoretically examined in the limit of dilute solutions. It is important to remark that $\left(D_{i j}\right)_{0} /\left[C_{i}\left(D_{i i}\right)_{0}\right]$ and $\left(L_{12}\right)_{0} /\left(C_{1} C_{2}\right)$ differ from $\left(D_{i j}\right)_{V} /\left[C_{i}\left(D_{i i}\right)_{V}\right]$ and $\left(L_{12}\right)_{V} /\left(C_{1} C_{2}\right)$, respectively, even at infinite dilution. Negative values of the Onsager cross-term $\left(L_{12}\right)_{0}$ are predicted for solvated solutes, consistently with experimental results. For two solvated solutes with a large difference in size, the $-\left(L_{12}\right)_{0} /\left(L_{11}\right)_{0}$ term in $\left(D_{12}\right)_{0} /\left[C_{1}\left(D_{11}\right)_{0}\right]$ cannot be neglected because it has a magnitude comparable to $\mu_{12} / \mu_{11}$. Correspond- 
ingly, $-\left(L_{12}\right)_{0} /\left(L_{22}\right)_{0}$ becomes negligible with respect to $\mu_{21} / \mu_{22}$ in $\left(D_{21}\right)_{0} /\left[C_{2}\left(D_{22}\right)_{0}\right]$. These predictions are consistent with experimental results. Excluded-volume interactions, which have been introduced through the McMillan-Mayer thermodynamic framework, are predicted to prevail with respect to preferential solvation for large globular solutes.

Acknowledgment. The author thanks John G. Albright for critical useful comments and Monika I. Antczak for stimulating discussions. This work was supported by the ACS Petroleum Research Fund (47244-G4) and TCU Research and Creative Activity Funds.

\section{Appendix A}

The isothermal and isobaric change in the Helmoholtz free energy, $A$, for an open system is given by

$$
\mathrm{d} A=-p \mathrm{~d} V+\sum_{i=0}^{N} \mu_{i} \mathrm{~d} n_{i}
$$

where $p$ is the pressure, $V$ is the volume, and $n_{i}$ is the number of moles of component $i$. By using $\mathrm{d} V=\sum_{i}^{N}=0 \bar{V}_{i} \mathrm{~d} n_{i}$, eq A1 becomes

$$
\mathrm{d} A=-\left(p-\mu_{0} / \bar{V}_{0}\right) \mathrm{d} V+\sum_{i=1}^{N}\left[\mu_{i}-\left(\bar{V}_{i} / \bar{V}_{0}\right) \mu_{0}\right] \mathrm{d} n_{i}
$$

The free energy of this multicomponent system can be compared to that of a pure reference solvent system with molar volume $\operatorname{vin} V_{0}$ and occupying the same total volume, $V$ :

$$
\mathrm{d} A^{*}=-\left(p-\mu_{0}^{*} / \bar{V}_{0}\right) \mathrm{d} V
$$

By subtracting eq A3 from eq A2, we obtain

$$
\mathrm{d} \tilde{A}=-\Pi \mathrm{d} V+\sum_{i=1}^{N}\left(\mu_{i}-\left(\bar{V}_{i} / \bar{V}_{0}\right) \mu_{0}\right) \mathrm{d} n_{i}
$$

where the difference $\tilde{A} \equiv A-A^{*}$ is the change in free energy when solvent molecules are replaced by solute molecules at constant volume and $\Pi=-\left(\mu_{0}-\mu_{0}^{*}\right) / \bar{V}_{0}$ is the osmotic pressure. Equation A4 shows that a liquid mixture of $N+1$ components can be equivalently treated as a compressible fluid consisting of $N$ effective components with pressure $\Pi$ and chemical potentials $\left.\tilde{\mu}_{i} \equiv \mu_{i}-\bar{V}_{i} / \bar{V}_{0}\right) \mu_{0}$. The $\tilde{\mu}_{i}$ 's are denoted as effective chemical potentials.

\section{Appendix B}

Experimental solvent-frame ternary diffusion coefficients have been used to extract chemical-potential derivatives, $\mu_{12}$ and $\mu_{21}{ }^{24}$ Equivalently, it will be now shown that experimental volumeframe diffusion coefficients can be used to extract the chemicalpotential derivative: $\tilde{\mu}_{12}=\tilde{\mu}_{21}$. For a ternary system, eq 12 becomes

$$
\left[\begin{array}{ll}
\left(D_{11}\right)_{V} & \left(D_{12}\right)_{V} \\
\left(D_{21}\right)_{V} & \left(D_{22}\right)_{V}
\end{array}\right]=\left[\begin{array}{ll}
\left(L_{11}\right)_{V} & \left(L_{12}\right)_{V} \\
\left(L_{21}\right)_{V} & \left(L_{22}\right)_{V}
\end{array}\right]\left[\begin{array}{ll}
\tilde{\mu}_{11} & \tilde{\mu}_{12} \\
\tilde{\mu}_{21} & \tilde{\mu}_{22}
\end{array}\right]
$$

Equation $\mathrm{B} 1$ can be solved with respect to $\tilde{\mu}_{12}$, yielding

$$
\tilde{\mu}_{12}=\frac{\tilde{\mu}_{11}\left(D_{12}\right)_{V}-\tilde{\mu}_{22}\left(D_{21}\right)_{V}}{\left(D_{11}\right)_{V}-\left(D_{22}\right)_{V}}
$$

Equation B2 can be used to determine $\tilde{\mu}_{12}$ provided that $\tilde{\mu}_{11}$ and $\tilde{\mu}_{22}$ are known. After the $\tilde{\mu}_{i j}$ matrix has been determined, the $\left(L_{i j}\right)_{V}$ matrix can be also calculated upon the inversion of eq B1. It is important to remark that the independent determi- nation of $\tilde{\mu}_{11}$ and $\tilde{\mu}_{22}$ is difficult in general. However, if the concentration of one solute, say $C_{1}$, is small compared to that of the other solute, $C_{2}$, we obtain $\tilde{\mu}_{11} / R T=1 / C_{1}$ and $\tilde{\mu}_{22}=$ $\tilde{\mu}_{22} *$, where $\tilde{\mu}_{22} *$ is the thermodynamic factor of the corresponding binary system. Thermodynamic data on binary systems are, by far, more accessible and available than those on ternary systems. Thus, eq B2 can be used to determine $\tilde{\mu}_{12}$ as a function of $C_{2}$ in the limit of small $C_{1}$. This approach could be used to probe excluded-volume interactions in colloid-polymer suspensions. ${ }^{29,30}$

\section{Appendix C}

Here, it is shown that $\nabla \tilde{\mu}_{i}=\nabla \mu_{i}+v_{i} \nabla \mu_{0}$ (with $\left.i=1,2, \ldots, N\right)$ is the appropriate thermodynamic driving force for a diffusion process examined with respect to the free-solvent reference frame. By inserting $\left(J_{i}\right)_{0}=\left(J_{i}\right)_{0}-\left(C_{i} / C_{0}\right)\left(J_{0}\right) \hat{0}$ into eq 2, we obtain

$$
-T \sigma=\sum_{i=1}^{N}\left[\left(J_{i}\right)_{0}^{\hat{0}}-\left(C_{i} / C_{0}\right)\left(J_{0}\right)_{0}\right] \nabla \mu_{i}
$$

Because $\hat{C}_{0}=C_{0}-\sum_{i}^{N}={ }_{1} v_{i} C_{i}$, we can also write

$$
\left(J_{0}\right)_{0}=\sum_{i=1}^{N} v_{i}\left(J_{i}\right)_{0}
$$

By inserting eq $\mathrm{C} 2$ into eq $\mathrm{C} 1$, we obtain

$$
\begin{aligned}
& -T \sigma=\sum_{i=1}^{N}\left[\left(J_{i}\right)_{0}-\left(C_{i} / C_{0}\right) \sum_{j=1}^{N} v_{j}\left(J_{j}\right)_{0}\right] \nabla \mu_{i}=\sum_{i=1}^{N}\left(J_{i}\right)_{0}\left(\nabla \mu_{i}+\right. \\
& \left.v_{i} \nabla \mu_{0}\right)=\sum_{i=1}^{N}\left(J_{i}\right)_{0} \nabla \hat{\mu}_{i}
\end{aligned}
$$

\section{References and Notes}

(1) Cussler, E. L. Diffusion, 2nd ed.; Cambridge Univ. Press: Cambridge, 1997.

(2) Crank, J. The Mathematics of Diffusion; Oxford at the Clarendon Press: Oxford, 1956.

(3) Tyrrell, H. J. V.; Harris, K. R. Diffusion in Liquids; Butterworths: London, 1984.

(4) Bird, R. B. AIChE J. 2003, 50, 273-287.

(5) Dunlop, P. J.; Gosting, L. J. J. Am. Chem. Soc. 1955, 77, 52385249.

(6) Albright, J. G.; Annunziata, O.; Miller, D. G.; Paduano, L.; Pearlstein, A. J. J. Am. Chem. Soc. 1999, 121, 3256-3266.

(7) Tan, C.; Albright, J. G.; Annunziata, O. J. Phys. Chem. B 2008 , $112,4967-4974$

(8) Annunziata, O.; Paduano, L.; Pearlstein, A. J.; Miller, D. G.; Albright, J. G. J. Phys. Chem. B 2006, 110, 1405-1415.

(9) Vergara, A.; Capuano, F.; Paduano, L.; Sartorio, R. Macromolecules 2006, 39, 4500-4506.

(10) Vergara, A.; Paduano, L.; Sartorio, R. J. Phys. Chem. B 2001, 105, 328-334.

(11) Vergara, A.; Paduano, L.; Sartorio, R. Phys. Chem. Chem. Phys. 2001, 3, 4340-4345.

(12) Jakupi, P.; Halvorsen, H.; Leaist, D. G. J. Phys. Chem. B 2004, 108, 7978-7985.

(13) Halvorsen, H. C.; Leaist, D. G. Phys. Chem. Chem. Phys. 2004, 6, $3515-3523$.

(14) Leaist, D. G. J. Phys. Chem. 1989, 93, 474-479.

(15) Wu, J. Q.; Albright, J. G.; Miller, D. G. J. Phys. Chem. 1994, 98 , 13054-13057.

(16) Cussler, E. L.; Lightfoot, E. N. J. Phys. Chem. 1965, 69, 11351144.

(17) Cussler, E. L.; Lightfoot, E. N. J. Phys. Chem. 1965, 69, 28752879.

(18) Gosting, L. J. Adv. Protein Chem. 1956, 11, 429-554.

(19) Miller, D. G. J. Phys. Chem. 1959, 63, 570-578.

(20) Vitagliano, V.; Sartorio, R. J. Phys. Chem. 1970, 74, 2949-2956.

(21) Miller, D. G.; Vitagliano, V.; Sartorio, R. J. Phys. Chem. 1986, 90, 1509-1519.

(22) Price, P. E.; Romdhane, I. H. AIChE J. 2003, 49, 309-322. 
(23) Ventras, J. S.; Ventras, C. M. Ind. Eng. Chem. Res. 2005, 44, 11121119.

(24) Ventras, J. S.; Ventras, C. M. Ind. Eng. Chem. Res. 2007, 46, 34223428 .

(25) Annunziata, O.; Paduano, L.; Pearlstein, A. J.; Miller, D. G.;

Albright, J. G. J. Am. Chem. Soc. 2000, 122, 5916-5928.

(26) Onsager, L. Ann. N.Y. Acad. Sci. 1945, 46, 241-265.

(27) Bearman, R. J. J. Phys. Chem. 1961, 65, 1961-1968.

(28) Zielinski, J. M.; Hanley, B. F. AIChE J. 1999, 45, 1-12.

(29) Lekkerkerker, H. N. W.; Poon, W. C. K.; Pusey, P. N.; Stroobants, A.; Warren, P. B. Europhys. Lett. 1992, 20, 559-564.

(30) Ilett, S. M.; Orrock, A.; Poon, W. C. K.; Pusey, P. N. Phys. Rev.

E 1995, 51, 1344-1352.

(31) McMillan, W. G.; Mayer, J. E. J. Chem. Phys. 1945, 13, 276-305.

(32) De Groot, S. R.; Mazur, P. Non-Equilibrium Thermodynamics; Elsevier: Amsterdam, 1962.

(33) Miller, D. G. Chem. Rev. 1960, 60, 15-37.

(34) Kirkwood, J. G.; Baldwin, R. L.; Dunlop, P. J.; Gosting, L. J.; Kegeles, G. J. Chem. Phys. 1960, 33, 1505-1513.

(35) Woolf, L. A.; Miller, D. G.; Gosting, L. J. J. Am. Chem. Soc. 1962,

$84,317-331$.

(36) Onsager, L. Phys. Rev. 1931, 37, 405-426.

(37) Dunlop, P. J.; Gosting, L. J. J. Phys. Chem. 1959, 63, 86-93.

(38) Hill, T. J. Chem. Phys. 1959, 30, 93-97.
(39) Van Solms, N.; Anderson, C. O.; Blanch, H. W.; Prausnitz, J. M. AIChE J. 2002, 48, 1292-1300.

(40) Lomakin, A.; Asherie, N.; Benedek, G. B. J. Chem. Phys. 1996, 104, 1646-1656.

(41) Xu, H.; Friedman, H. L.; Raineri, F. O. J. Sol. Chem. 1991, 20, 739-773.

(42) Felderhof, B. U. J. Chem. Phys. 2003, 118, 11326-11334.

(43) Kops-Werkhoven, M. M.; Vrij, A.; Lekkerkerker, H. N. W. J. Chem. Phys. 1983, 78, 2760-2763.

(44) Lightfoot, E. N. E.; Cussler, E. L.; Rettig, R. L. AIChE J. 1962, 8, $708-709$.

(45) Albright, J. G. J. Phys. Chem. 1969, 73, 1280-1286.

(46) Tanford, C. Physical Chemistry of Macromolecules; Wiley: New York, 1961.

(47) Lebowitz, J. L. Phys. Rev. A 1964, 133, 895-899.

(48) Timasheff, S. N. Proc. Natl. Acad. Sci.U. S. A. 1998, 95, 73637367.

(49) Parsegian, V. A.; Rand, R. P.; Rau, D. C. Proc. Natl. Acad. Sci.U. S. A. 2000, 97, 3987-3992.

(50) Record, M. T., Jr.; Anderson, C. F. Biophys. J. 1995, 68, 786794.

(51) Annunziata, O.; Paduano, L.; Albright, J. G. J. Phys. Chem. B 2007, 111, 10591-10598.

JP803995N 